\title{
High-resolution rapid neonatal whole-body composition using 3.0 Tesla chemical shift magnetic resonance imaging
}

\author{
Jonathan P. Dyke ${ }^{1}$, Amanda C. Garfinkel ${ }^{2}$, Alan M. Groves ${ }^{2}$ and Arzu Kovanlikaya ${ }^{1}$
}

BACKGROUND: To evaluate a whole-body rapid imaging technique to calculate neonatal lean body mass and percentage adiposity using 3.0 Tesla chemical shift magnetic resonance imaging (MRI).

METHODS: A 2-Point Dixon MRI technique was used to calculate whole-body fat and water images in term $(n=10)$ and preterm $(n=15)$ infants.

RESULTS: Chemical shift images were obtained in $42 \mathrm{~s}$. MRI calculated whole-body mass correlated closely with measured body weight $\left(R^{2}=0.87 ; \quad P<0.001\right)$. Scan-rescan analysis demonstrated a $95 \%$ limit of agreement of $1.3 \%$ adiposity. Preterm infants were born at a median of 25.7 weeks' gestation with birth weight $840 \mathrm{~g}$. At term-corrected age, former preterm infants were lighter than term-born controls, 2,519 vs. 3,094 g regressing out age and group as covariates $(P=0.005)$. However, this was not because of reduced percentage adiposity $26 \%$ vs. $24 \% \quad(P=0.28)$. At termcorrected age, former preterm infants had significantly reduced lean body mass compared with that of term-born controls 1,935 vs. $2,416 \mathrm{~g}(P=0.002)$.

CONCLUSION: Rapid whole-body imaging for assessment of lean body mass and adiposity in term and preterm infants is feasible, accurate, and repeatable. Deficits in whole-body mass in former preterm infants at term-corrected age are due to reductions in lean body mass not due to differences in adiposity.

\section{A} dequate nutrition during early life is crucial for proper neurologic and cognitive development, and emerging evidence suggests that nutritional status during this critical developmental period also influences cardiometabolic health through childhood and adulthood (1-3).

Despite neonatologists' efforts to nourish preterm infants in such a way that their growth trajectories mirror that which would naturally occur in utero, there is substantial evidence to suggest that when preterm infants reach term-corrected age (TCA), their body composition differs markedly from that of their healthy, term-born counterparts. Preterm infants at TCA, although lighter and shorter, may have higher body fat percentages $(3,4)$. These preterm-born infants at TCA appear to have a significant deficit in fat-free mass, suggesting that their overall decreased body weight reflects a lack of sufficient lean tissue accretion (3).

Efforts to optimize the nutritional care of preterm infants are limited by existing methods of growth-monitoring and body composition assessment in newborns. In current clinical practice, neonatal growth is tracked by plotting weight and length along growth curves, without consideration for the differential accumulation of fat vs. lean tissue mass. In research settings, methodologies such as skinfold thickness measurement, air-displacement plethysmography (ADP), and dual X-ray absorptiometry (DXA) have been used to distinguish fat vs. lean mass in newborns (5). The Cosmed (Pea Pod) system (Concord, CA) calculates fat mass and fatfree mass using an accurate scale, whereas body volume is calculated from air displacement in a chamber. The technique is quick and accurate, but gives no information on regional deposition of adipose tissue, similar to magnetic resonance imaging (MRI), and cannot be used when an infant is requiring respiratory support (6). DXA calculates both fat and fat-free mass using a dual energy X-Ray bone density measurement. Infants must be asleep while undergoing the procedure, and an overestimation of $12-30 \%$ of total body fat has been shown (7). As with ADP, DXA also does not give any information on regional fat deposition. A recent study also showed that, although ADP and DXA are highly correlated, significant differences in body composition are observed between the techniques (8).

Chemical-shift MRI is an imaging technology that takes advantage of the differential resonance frequencies of protons in fat vs. water, producing an image of fat vs. non-fat tissues in the body. The technique was introduced in 1984 by Dixon who produced water-only and fat-only images by shifting the MRI read-out gradient to produce in-phase (IP) and $180^{\circ}$ outof-phase (OOP) images (9). At the time, a lack of $B_{0}$ homogeneity and increased scan times made the technique prohibitive in the clinic. However, multiple advances in hardware, pulse sequences, and post-processing have now made this technique very accurate and rapid $(10,11)$. Chemical-shift MRI represents a non-invasive, radiation-free

${ }^{1}$ Department of Radiology, Weill Cornell Medicine, New York, New York; ${ }^{2}$ Department of Pediatrics, Weill Cornell Medicine, New York, New York. Correspondence: Jonathan P. Dyke (jpd2001@med.cornell.edu)

Received 12 July 2017; accepted 17 October 2017; advance online publication 20 December 2017. doi:10.1038/pr.2017.294 
method of body composition assessment that can potentially be implemented into the routine nutritional management of preterm neonates. In the present study, we endeavored to demonstrate that chemical-shift MRI is a rapid, detailed, and robust method of body composition analysis that can be feasibly implemented into the routine clinical care of newborns. Furthermore, we aimed to show that chemicalshift MRI has the sensitivity to detect differential growth patterns in preterms at TCA vs. term controls.

\section{METHODS}

\section{Subjects}

Twenty-five infants were enrolled in the study (10 male and 15 female subjects, median (range) postnatal age 69 (1-138) days, median (range)-corrected gestational age 39.4 (36.4-44.7) weeks), with 15 former preterm and 10 term infants (Table 1). Preterm infants were born at a median (range) of 25.7 (24.1-29.3) weeks' gestation with birth weight $840(590-1,210)$ g. All research scans were acquired under a protocol approved by our Institutional Review Board, and informed consent was obtained from parents of all subjects.

\section{MRI Preparation and Clinical Monitoring}

Infants were prepared for the MRI examination using a "feed and wrap" technique developed and previously described by one of the authors (A.M.G.) and colleagues (12). Briefly, infants were fed and allowed to fall into a natural sleep without sedation or anesthesia. They were then provided three layers of hearing protection (soft foam earplugs, plastic ear muffs, and blanket padding), securely swaddled with blankets, and placed in a MedVac vacuum immobilization pack, which contains polystyrene granules and gently molds to the infant's form.

MRI-compatible ECG leads were placed on the chest and a pulse oximeter was placed on the foot. The infant's heart rate and oxygen saturation were monitored through the duration of the scan. Signals of an infant waking from sleep, such as loss of oxygen tracing or persistent increase in heart rate, prompted examiners to enter the MRI room to check on the infant as soon as possible, and the scan was stopped if the infant did not quickly and comfortably fall back to sleep.

\section{MRI Acquisition}

High-resolution ( $1 \mathrm{~mm}^{3}$ isotropic) whole-body fat and water images were acquired on a 3.0 Tesla General Electric MRI scanner (GE Healthcare, Milwaukee, WI) using the adult Head/Neck 48-Channel Array coil and the Liver Acquisition with Volume Acceleration flex (LAVA-Flex) pulse sequence. The head and neck coil provided adequate full-body neonatal coverage for a fully swaddled infant. A coronal three-dimensional slab was acquired during free-breathing with 160 images using the following parameters: a repetition time/ echo time of $4.0 \mathrm{~ms} / 1.7 \mathrm{~ms}$, a flip angle of $12^{\circ}, 48 \mathrm{~cm}$ field of view, and a $288 \times 288$ matrix reconstructed to $512 \times 512$ yielding a total scan time of only $42 \mathrm{~s}$. For all examinations, specific absorption rate levels were within allowed FDA limits $(1.48 \pm 0.35 \mathrm{~W} / \mathrm{kg})(13,14)$. The LAVA-Flex sequence acquires IP and OOP images in order to perform a 2-Point Dixon method to construct pure water-only (Figure 1a) and pure-fat only (Figure 1b) images (15). The equations used to create the water- (W) and fat- (F) only images on the scanner were: $W=\frac{1}{2}[\mathrm{IP}+\mathrm{OOP}], F=\frac{1}{2}[\mathrm{IP}-\mathrm{OOP}]$ (Note: correct when $\mathrm{W}>\mathrm{F})(6)$.

\section{MRI Analysis}

Quantitative fat fraction maps created by the scanner were not utilized as fat fractions greater than $100 \%$ were encountered,
Table 1. Demographic data are given for the 25 subjects along with calculated MRI and actual measured weights

\begin{tabular}{|c|c|c|c|c|c|c|c|}
\hline Subject & Age & Sex & $\begin{array}{l}\text { MRI1 Wt } \\
\text { (g) }\end{array}$ & $\begin{array}{l}\text { MRI2 Wt } \\
\text { (g) }\end{array}$ & $\begin{array}{l}\text { Actual } \\
\text { Wt (g) }\end{array}$ & $\begin{array}{l}\text { Birth } \\
\text { GA } \\
\text { (wks) }\end{array}$ & $\begin{array}{l}\text { CGA } \\
\text { (wks) }\end{array}$ \\
\hline 1 & 7 & $\mathrm{~F}$ & $3,173.8$ & a & $3,255.0$ & 41.0 & 42.0 \\
\hline 2 & 3 & M & $2,744.6$ & $2,695.1$ & $3,015.0$ & 37.0 & 37.4 \\
\hline 3 & 1 & M & $3,114.2$ & $3,128.5$ & $3,535.0$ & 39.0 & 39.1 \\
\hline 4 & 100 & $\mathrm{~F}$ & $2,169.0$ & $2,239.4$ & $2,080.0$ & 24.1 & 38.7 \\
\hline 5 & 72 & $\mathrm{~F}$ & $1,867.6$ & $1,720.1$ & $2,014.0$ & 25.7 & 37.0 \\
\hline 6 & 105 & $\mathrm{~F}$ & $2,283.9$ & $2,371.1$ & $2,625.0$ & 24.1 & 39.3 \\
\hline 7 & 98 & $M$ & $2,068.5$ & $2,033.0$ & $2,600.0$ & 25.0 & 39.0 \\
\hline 8 & 85 & $\mathrm{~F}$ & $2,723.3$ & a & $2,585.0$ & 25.7 & 37.9 \\
\hline 9 & 92 & M & $2,679.2$ & $2,890.5$ & $2,720.0$ & 25.7 & 38.9 \\
\hline 10 & 138 & $M$ & $2,678.0$ & $2,675.8$ & $2,800.0$ & 25.0 & 44.7 \\
\hline 11 & 6 & $\mathrm{~F}$ & $2,775.3$ & $2,726.1$ & $2,720.0$ & 37.6 & 38.6 \\
\hline 12 & 66 & $\mathrm{~F}$ & $2,112.6$ & $\mathrm{~b}$ & $2,355.0$ & 27.0 & 36.4 \\
\hline 13 & 67 & $\mathrm{~F}$ & $2,400.0$ & $2,379.6$ & $2,445.0$ & 27.0 & 36.6 \\
\hline 14 & 69 & $\mathrm{~F}$ & $2,309.2$ & $2,362.2$ & $2,270.0$ & 27.6 & 37.4 \\
\hline 15 & 96 & M & $2,992.4$ & c & $3,010.0$ & 25.4 & 39.1 \\
\hline 16 & 11 & M & $3,537.2$ & $3,556.2$ & $4,050.0$ & 40.4 & 42.1 \\
\hline 17 & 114 & $\mathrm{~F}$ & $2,259.7$ & $2,201.9$ & $2,540.0$ & 24.9 & 41.0 \\
\hline 18 & 101 & $\mathrm{~F}$ & $3,451.2$ & $3,470.3$ & $3,440.0$ & 25.7 & 40.1 \\
\hline 19 & 102 & M & $2,968.3$ & c & $3,220.0$ & 25.7 & 40.3 \\
\hline 20 & 91 & $\mathrm{~F}$ & $2,822.5$ & c & $2,845.0$ & 29.3 & 42.1 \\
\hline 21 & 5 & $\mathrm{~F}$ & $2,161.0$ & $2,110.7$ & $2,285.0$ & 37.4 & 38.1 \\
\hline 22 & 4 & $\mathrm{~F}$ & $3,172.7$ & $3,165.7$ & $3,290.0$ & 38.1 & 38.7 \\
\hline 23 & 1 & $\mathrm{~F}$ & $4,128.4$ & $b$ & $3,965.0$ & 39.6 & 39.7 \\
\hline 24 & 3 & $M$ & $3,189.5$ & $3,292.8$ & $3,580.0$ & 39.3 & 39.7 \\
\hline 25 & 1 & M & $2,944.2$ & $2,953.5$ & $3,345.0$ & 39.6 & 39.9 \\
\hline
\end{tabular}

reflecting inaccuracies in the manufacturer's algorithm. Macros were written in Image (Rasband WS; NIH) to create 32-bit fat fraction maps. An image calculation of $F /(F+W)$ images was performed, resulting in each voxel having a value $0-100 \%$ (Figure 1c). An additional ImageJ macro was written (Supplementary Appendix S1 online) to cleanly segment the whole body from the fat fraction map that included noise voxels outside the infant. The water and fat images were first converted to a binary mask (Figure 2a,b) including all voxels with a signal intensity $\geq 5 \%$ of the stack maximum. This signal intensity cutoff was kept constant for all subjects. The water and fat masks were summed, and a single erosion and dilation step was performed. Specifically, in ImageJ, a $3 \times 3$ kernel traverses the image replacing each pixel with the minimum voxel intensity (erosion) or the maximum voxel intensity (dilation) in the neighborhood. This aids in removing noise voxels outside the body, which may be a result of motion artifacts. The summed wholebody mask (Figure 2c) was multiplied by the calculated fat fraction map (Figure 1c) to produce the clean map as shown in Figure 2d. The map was cropped to eliminate any spurious voxels outside the body.

A histogram was created with 100 bins categorizing all fat fraction voxels in the image. The total fat fraction (\%FF) represents the area under the histogram $\geq 50 \%$ fat normalized to the total histogram 

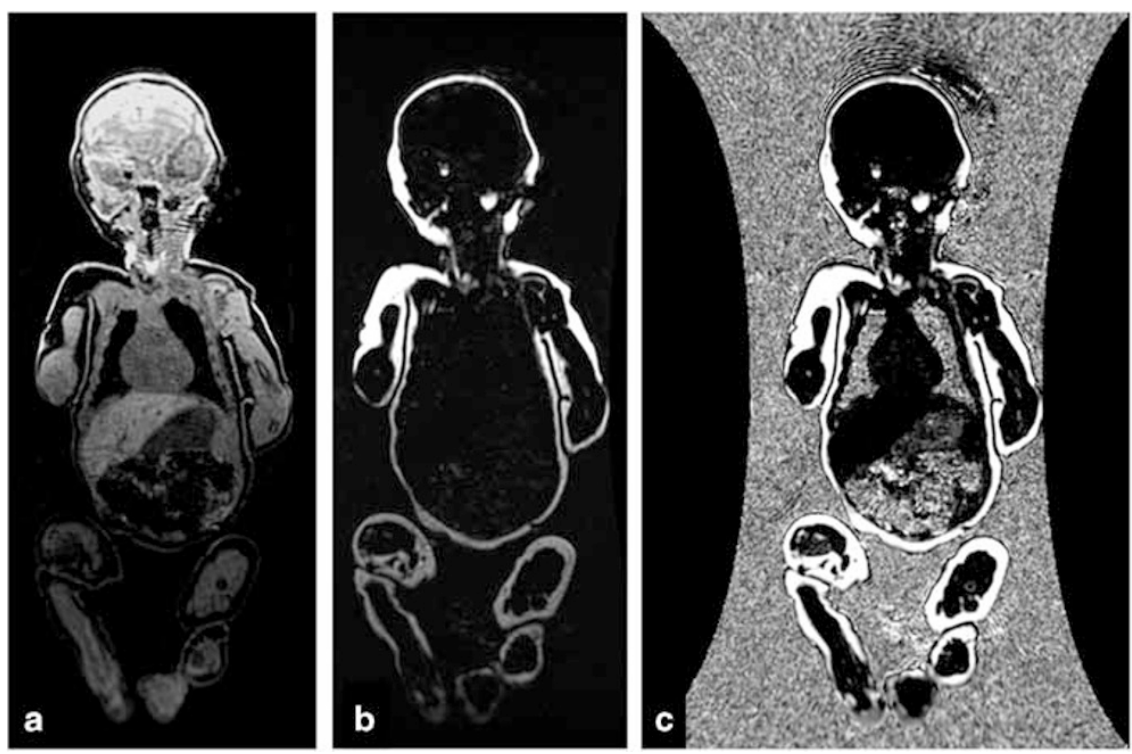

Figure 1. (a) Lava-Flex water-only image created as (IP+OP)/2. (b) Lava-Flex fat-only image created as (IP-OP)/2. (c) Calculated ImageJ 32-bit fat fraction image as fat/(water+fat). IP, in-phase; Lava-Flex, liver acquisition with volume acceleration flex; OP, out-of-phase.

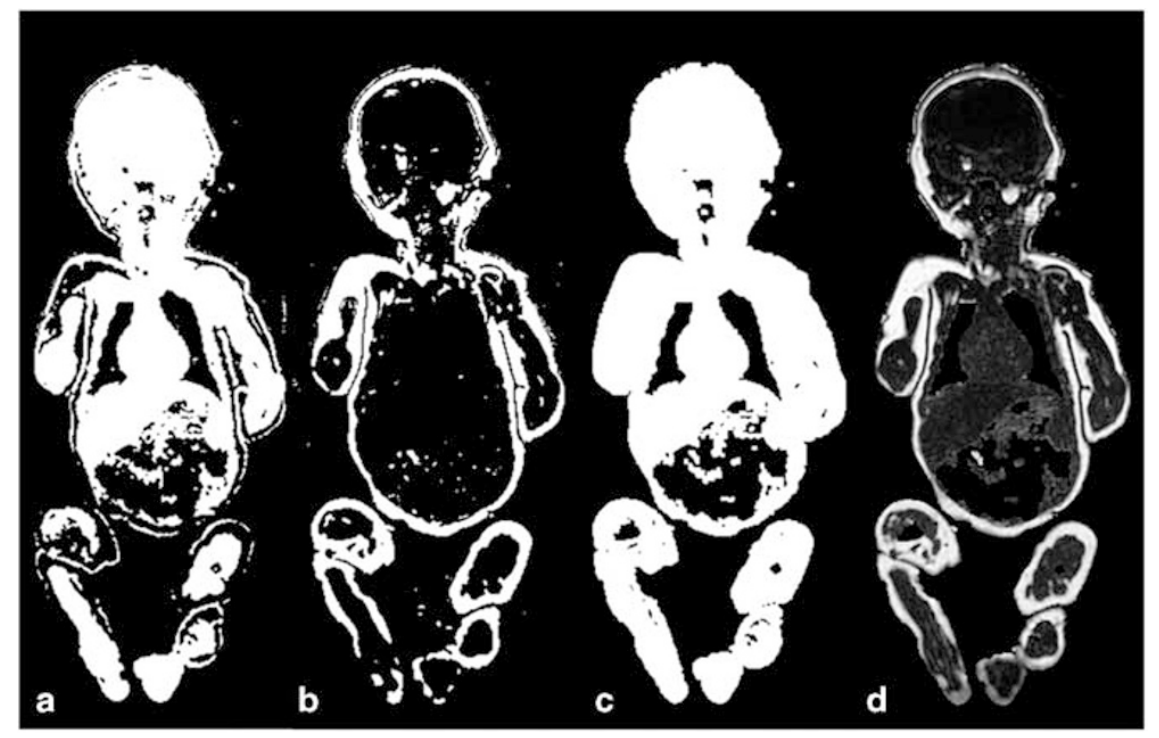

Figure 2. (a) The segmented water-only binary mask containing voxels $\geq 5 \%$ of the series. (b) The segmented fat-only binary mask containing voxels $\geq 5 \%$ of the series. (c) The combined total body binary mask including an erosion and dilation step. (d) The final fat fraction map is then the product of Figure $1 \mathrm{c}$ and Figure $2 \mathrm{c}$.

area. The total body weight was estimated using equation 1 :

$$
\begin{aligned}
\text { MRI Weight }= & \text { Total Volume }(\mathrm{cc})[\% \mathrm{FF} * 0.90(\mathrm{~g} / \mathrm{cc}) \\
& +(1-\% \mathrm{FF}) * 1.06(\mathrm{~g} / \mathrm{cc})]
\end{aligned}
$$

The total number of voxels in the binary mask was multiplied by the voxel size (cc) to get the total volume. This was multiplied by the MRI-determined $\% W$ and $\% F$ fractions and their respective tissue densities to yield the MRI-determined weight $(15,16)$. The lean body mass was calculated as the total volume $(\mathrm{cc}) \times(1-\% \mathrm{FF}) \times 1.06(\mathrm{~g} / \mathrm{cc})$.

\section{Post-hoc Correction for Phase Mismatch}

A phase mismatch artifact was observed on seven subjects primarily in the extremities. The scanner did not allow for rectangular or multiple shim volumes, so the air-tissue interface was quite prominent in some infants if the arms or legs were not able to be swaddled close to the body. An example of a phase mismatch in the lower extremities may be seen as a false hyperintensity in the legs in the fat fraction image (Figure 3a). The fat fraction map is a parametric map with values in each voxel ranging from 0 to $100 \%$. If the fat and water components are swapped, then the simple calculation of 100 -Voxel\% needs to be performed in that specific region. A procedure was developed within ImageJ that was robust to the region of interest (ROI) chosen and was able to correct this artifact.

The image stack was duplicated and the stack threshold adjusted to create a black background that included only image data in a binary mask greater than $0.4 \%$ fat fraction. An ROI was loosely drawn outlining the affected region on each slice. It is of note to mention that an exact tracing was not required, which would require an additional level of expertise as well as additional time. The resulting 


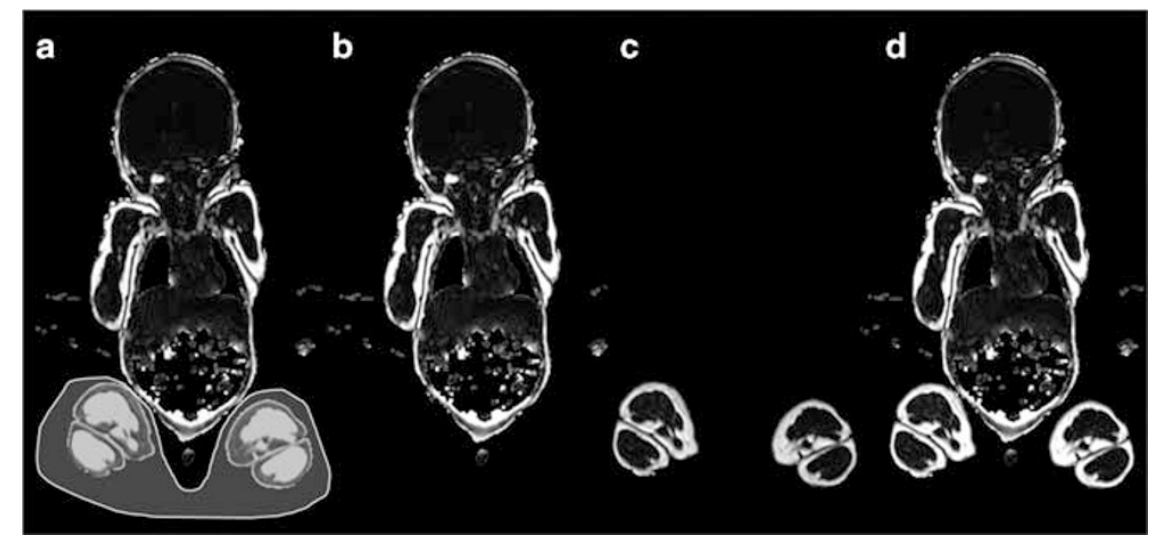

Figure 3. An example of a phase mismatch in the lower extremities is shown in the fat fraction image. Note the false hyperintense assignment of fat in the legs. (a) An ROI is loosely drawn outlining the affected region noting that an exact tracing is not required. (b) A binary map of this ROI is propagated throughout the affected slices and multiplied by the fat fraction image to remove the region. (c) The correction of $100-\mathrm{Voxel} \%$ is performed just on the legs to create the phase-corrected image. (d) The corrected fat fraction map is then made by summing (b,c). ROI, region of interest.

binary map was propagated throughout the stack and multiplied by the fat fraction image to remove the region (Figure $3 \mathbf{b}$ ). A correction of $100-$ Voxel\% was performed to create the phase-corrected image as shown on the legs (Figure 3c). The final corrected fat fraction map was then made by summing the images shown in Figure $3 \mathbf{b}, \mathbf{c}$.

\section{Statistical Analyses}

A Pearson's correlation coefficient $(R)$ was determined from the linear regression analysis comparing MRI and actual measures of weight. The statistical significance of the regression fit was determined using a two-tailed $t$-test with $P<0.05$. A multiple regression was used to account for age and group as covariates when comparing lean body mass between preterm $(n=15)$ and term $(n=10)$ infants. The Bland-Altman difference test was performed to determine whether the $95 \%$ limits of agreement of adiposity on repeat testing.

\section{RESULTS}

External Validation of Chemical-Shift MRI Against Body Weight The calculated MRI weight was correlated with the actual body weight using the Pearson's correlation coefficient $\left(R^{2}=0.87, \quad P<0.001 ;\right.$ Figure 4). The linear regression yielded equation 2 :

$$
\begin{aligned}
& \text { Actual Measured Weight }(\mathrm{g}) \\
& \quad=0.96 * \text { MRI Calculated Weight }(\mathrm{g})+256.4 \mathrm{~g}
\end{aligned}
$$

The resulting regression slope was nearly unity, supporting the validity of MRI measures. At term-corrected age, former preterm infants were lighter than term-born controls (median (range) 2,519 (1,860-3,451)g vs. 3,094 (2,161-4,128)g, yielding a significant difference when regressing out age and group as covariates $(P=0.005)$.

\section{Scan-Rescan Chemical-Shift MRI Assessment of Total Fat Fraction}

A scan-rescan analysis was performed in 18 of 25 subjects (Table 1). Scan-rescan could not be performed if only one sequence was obtained $(n=2)$, if severe phase mismatch artifacts were present $(n=3)$, or if severe motion $(n=2)$ occurred when the infant awoke. One scan was also excluded

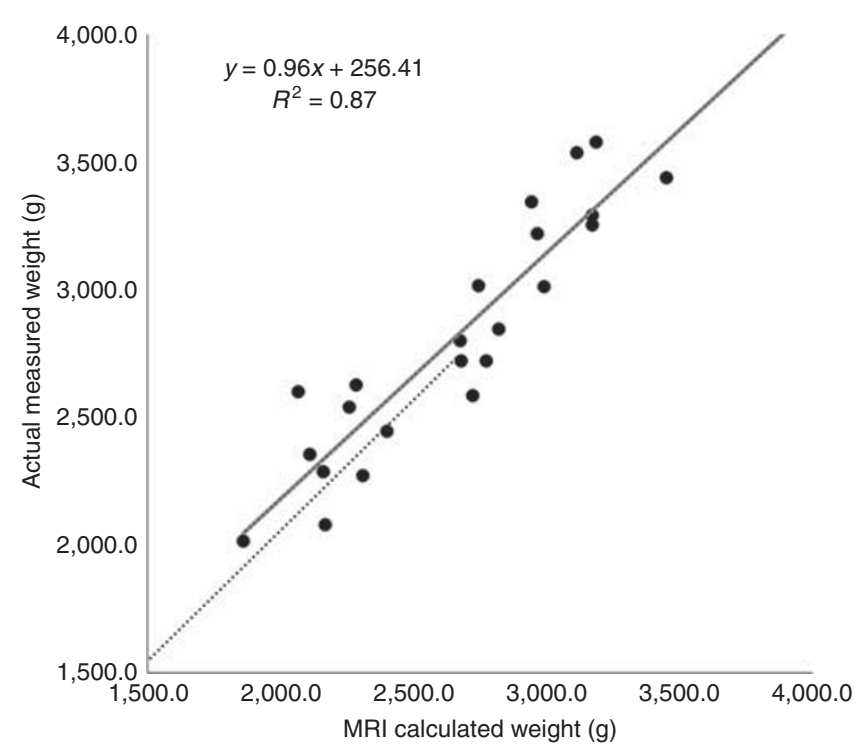

Figure 4. Correlation of the MRI-determined body weight (calculated using equation 2 ) is shown against the actual measured body weight performed on a digital scale at the time of the MRI scan $\left(R^{2}=0.87\right)$. The line of unity is shown, which duly follows the distribution of the subjects and illustrates an accuracy in the technique. MRI, magnetic resonance imaging.

as it required a threshold on the water and fat images of 3\% maximum value, given low signal-to-noise ratios in the extremities due to coil positioning. The result for the BlandAltman test between consecutive whole-body fat fraction acquisitions is shown in Figure 5. The dashed line displays the mean difference in adiposity between repeat examinations $(0.37 \%)$ with SD of 0.65 . The $95 \%$ limits of agreement $(1.96 \times \mathrm{SD})$ were, therefore, $1.3 \%$.

Body Composition in Preterm at TCA vs. Full-Term Subjects Figure 6 shows a whole-body histogram for a preterm infant with a total fat fraction of $26.0 \%$. Data on body mass and 


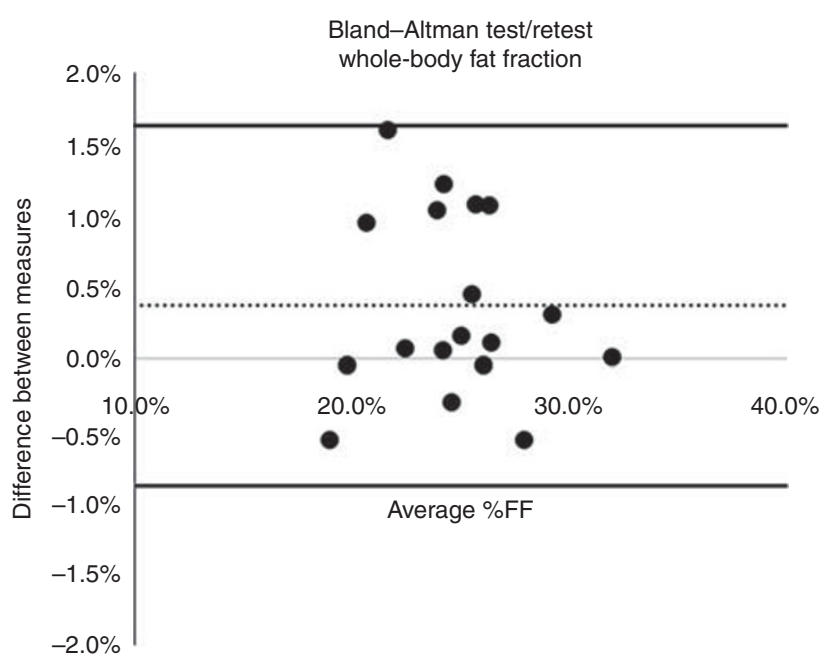

Figure 5. The result for the Bland-Altman test between consecutive whole-body fat fraction acquisitions is shown $(n=18)$. The dashed line displays the mean $(0.37 \%)$ variability between scans within a range of $-0.89-1.62 \%$ of the $95 \%$ limits of agreement.

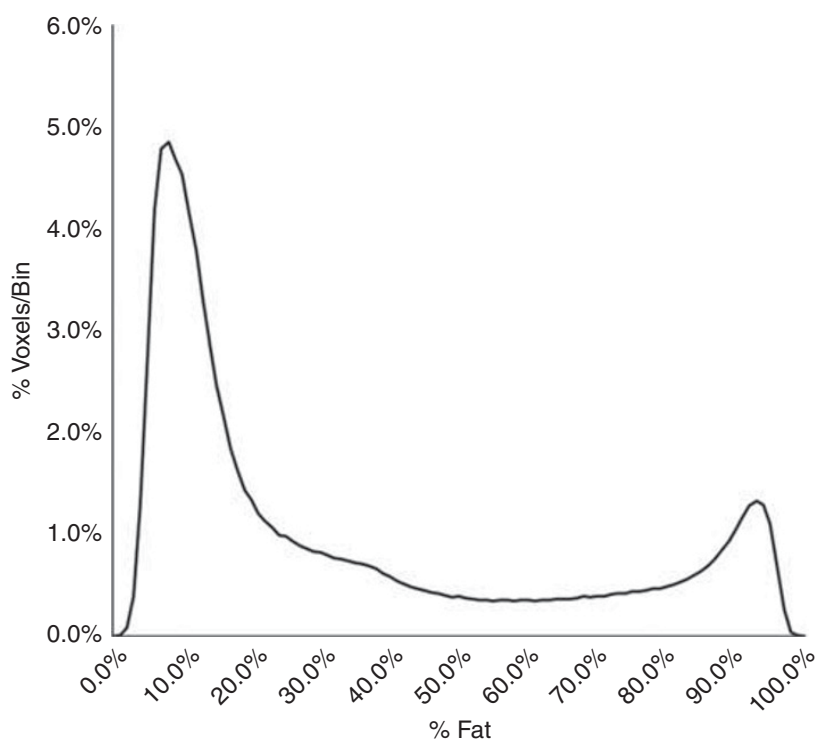

Figure 6. The whole-body fat fraction histogram of a preterm infant. Adipose tissue is categorized as a percent fat $\geq 50 \%$ and voxels are summed to yield a fat fraction of $26.0 \%$ in this subject.

composition in former preterm infants compared with termborn controls are shown in Table 2. The decrease in MRIdetermined body weight was not due to reduced percentage adiposity (median (range) $26 \quad(18.9-33.4) \%$ vs. 24 $(18.7-29.5) \% \quad(P=0.28))$. Likewise, the absolute MRIdetermined fat mass showed no significant difference in former preterm (median (range) $593.6(449.4-899.3) \mathrm{g}$ ) vs. term infants (median (range) 690.9 (436.2-934.0)g; $P=0.19$ ).

A multivariate regression was performed of lean body mass in (17) preterm-born infants studied at TCA vs. (10) healthy term-born controls' regressing-out age (CGA) and group as covariates. The regression results demonstrated that there was a significant group change $(P=0.002)$ in lean body mass
Table 2. MRI-determined weight, adiposity, and body mass fractions are shown for term infants $(n=10)$ and former preterm infants $(n=15)$

\begin{tabular}{lccc}
\hline & Term & Former preterm & $P$ \\
\hline Body weight by MRI & $3,094.1 \pm 516.9 \mathrm{~g}$ & $2,518.5 \pm 429.7 \mathrm{~g}$ & 0.005 \\
Lean body mass by MRI & $2,416.5 \pm 344.4 \mathrm{~g}$ & $1,934.9 \pm 344.4 \mathrm{~g}$ & 0.002 \\
Absolute fat mass by MRI & $690.9 \pm 199.5 \mathrm{~g}$ & $593.6 \pm 142.0 \mathrm{~g}$ & 0.19 \\
Percentage adiposity by & $24.5 \pm 3.4 \%$ & $26.2 \pm 4.0 \%$ & 0.28 \\
MRI & & \\
\hline CGA, controls regressing out age; MRI, magnetic resonance imaging. \\
The $P$ value results from the group difference as determined using a multivariate \\
linear regression with CGA and group as covariates.
\end{tabular}

independent of age. At term-corrected age, former preterm infants had significantly reduced lean body mass compared with term-born controls (median (range) 1,935 (1,418-2,657)g vs. $2,416(1,735-3,047) \mathrm{g})$. Although these sample sizes are small, these initial findings demonstrate the proof of concept that chemical-shift MRI is a technique with sufficient sensitivity to detect growth and body composition differences in preterms at TEA compared with full-term controls.

\section{DISCUSSION}

To our knowledge, our study is the first description of an isotropic rapid whole-body chemical-shift 3.0 Tesla MRI assessment of lean body mass and whole-body adiposity specifically in newborns. Several prior studies have used differing MRI techniques to estimate fat deposition in infants and children. One study examined 35 infants (25 appropriate for gestational age and 10 growth-restricted) using a $\mathrm{T}_{1}$-Weighted sequence at 1.5 Tesla ( (ref. 18)). The technique used a $10-\mathrm{mm}$ slice thickness with a $30-\mathrm{mm}$ gap in the axial direction to cover the whole body (19). The study showed a reduction in total adipose tissue in growth-restricted infants due to a difference in subcutaneous tissue. Our study improved on these techniques by utilizing a rapid $1 \mathrm{~mm}^{3}$ isotropic resolution with no gaps. In addition, a $\mathrm{T}_{1}$-weighted technique does not truly separate fat and water, and absolute quantitation of adipose tissue is highly dependent on the thresholds chosen for analysis. In addition, the acquisition parameters chosen (repetition time, echo time, pulse sequence (gradient echo vs. spin echo)) may change the degree of $\mathrm{T}_{1}$-weighting and thereby alter the quantitative results. We used a 2-Point Dixon technique to accurately separate fat and water components, allowing for robust repeatability and comparison across subjects.

Another study examined whole-body adipose tissue using a Dixon MRI technique on 11 adults using a 1.5 Tesla MRI (20). Continuous table movement and a 5-mm slice thickness were recorded on the images. A similar masking technique was described as in our study but an estimation for partial volume contributions was required, given the large slice thickness. In addition, the total scan time was $12 \mathrm{~min}$ and required multiple $15 \mathrm{~s}$ breath holds at each bed position, which precludes use of this technique in newborns. Lastly, a recent study utilized a PROPELLER sequence with a $5-\mathrm{mm}$ slice thickness in 53 infants (21). This study was not performed on newborns but the infants had an average age of 67 days ranging from 22 to 
154 days. In addition, motion-free images in natural sleeping infants were achieved in only 36 of the 53 MRI scans. Likewise, the PROPELLER sequence took 7:24 min resulting in a 5-mm slice thickness.

In this current cohort of 25 infants (10 term and 15 preterm), chemical-shift MRI proved to be a rapid, accurate, and repeatable method of body composition analysis that may be feasible for implementation in routine nutritional management of preterm-born infants. Chemical-shift MRI is a robust method of assessing body composition. Body weight calculated by chemical-shift MRI was highly correlated with actual measured body weight, with an $R^{2}=0.87 \quad(P<0.001)$. Furthermore, these chemical-shift MR images can be used to quantify lean body mass and fat mass with high repeatability. Another advantage of chemical-shift MRI is its speed; the LAVA-Flex sequence utilized in this study takes only $42 \mathrm{~s}$. In comparison, studies using ADP have reported scan times of 3-5 min, whereas $T_{1}$-weighted MRI assessment of body composition has been reported to take $6 \mathrm{~min}$ or longer (22-25).

Chemical-shift MRI is not only robust, but also highly detailed. In the present study, we were able to visualize both total and regional adipose tissue depots at $1 \mathrm{~mm}^{3}$ isotropic resolution. This stands in great contrast to ADP, which measures whole-body fat mass but provides no information on fat distribution. In seeking to better understand the differential growth of preterm vs. full-term infants, it may be important to track not just whole-body fat accumulation but also the fat distribution, with such high resolution. Future studies using chemical-shift MRI can be further developed to assess regional fat depots.

Finally, chemical-shift MRI is preferable to other body composition-assessment tools because of its safety profile. Unlike DXA scanning, MRI involves no radiation. The "feed and wrap" technique allows acquisition of high-quality images with minimal motion artifact without the use of any sedation or anesthesia. In addition, although all infants in this study were clinically stable, chemical-shift MRI can be used to monitor growth in preterm infants of various clinical conditions; for example, chemical-shift MRI can be used in infants requiring respiratory support, whereas ADP is respiratory support-incompatible.

Despite these advantages, which render chemical-shift MRI an optimal tool for monitoring growth and body composition in newborns, the technique has some known limitations. The 2-point Dixon method assumes that the dominant signal in the water image is indeed water. A binary choice must be made to assign water or fat, which is difficult if the $\% \mathrm{FF}$ is near $50 \%$. Our method slightly but consistently underestimates total body weight, possibly related to difficulties with delineation of air spaces within the abdomen (Figure 2). There are also barriers to the widespread clinical implementation of MRI for this purpose. MRI scans are expensive, and MRI machinery and personnel are not always available in settings where preterm infants are receiving nutritional care. Furthermore, in many institutions, there may be a lack of exposure to the "feed and wrap" techniques required to scan an infant without anesthesia; clinical training may thus be required to make chemical-shift MRI feasible in routine clinical care.

Although the present study suggests that chemical-shift MRI represents a rapid, detailed, robust, and safe method of body composition analysis in newborns, there are limitations to the current study, including a small sample size. In addition, the scan-rescan protocol did not take the infant out of the scanner between runs, which would be unrealistic in clinical practice and wake up the child.

\section{Conclusions}

In summary, chemical-shift MRI represents a safe, rapid, robust, and detailed method of body composition analysis that holds potential to reform the way we monitor growth in newborns and to optimize the nutritional management of those infants born preterm. Although the current study has focused on the particular problem of altered growth and body composition in preterm infants, chemical-shift MRI can be similarly applied to the care of other neonatal populations with altered growth patterns, such as intrauterine growthrestricted infants and infants born to diabetic mothers. As growth and nutrition during the neonatal period are critical determinants of an infant's later development and health status, novel imaging methods such as chemical-shift MRI may allow clinicians to continue to advance strategies for optimally nourishing and monitoring growth in newborns.

\section{STATEMENT OF FINANCIAL SUPPORT}

No financial assistance was received in support of this study.

Disclosure: The authors declare no conflict of interest.

\section{REFERENCES}

1. Hsiao CC, Tsai ML, Chen CC, Lin HC. Early optimal nutrition improves neurodevelopmental outcomes for very preterm infants. Nutr Rev 2014;72:532-40.

2. Schneider N, Garcia-Rodenas CL. Early nutritional interventions for brain and cognitive development in preterm Infants: a review of the literature. Nutrients 2017;9:1-20.

3. Johnson MJ, Wootton SA, Leaf AA, Jackson AA. Preterm birth and body composition at term equivalent age: a systematic review and metaanalysis. Pediatrics 2012;130:640-9.

4. Daly-Wolfe KM, Jordan KC, Slater H, Beachy JC, Moyer-Mileur LJ. Midarm circumference is a reliable method to estimate adiposity in preterm and term infants. Pediatr Res 2015;78:336-41.

5. Rice MS, Valentine CJ. Neonatal body composition: measuring lean mass as a tool to guide nutrition management in the neonate. Nutr Clin Pract 2015;30:625-32.

6. McLeod G, Simmer K, Sherriff J, Nathan E, Geddes D, Hartmann P. Feasibility study: Assessing the influence of macronutrientintakes on preterm body composition, using air displacement plethysmography. J Ped Child Health 2015;51:862-9.

7. Rigo J, Nyamugabo K, Picuad JC, Gerard P, Pieltain C, De Curtis M. Reference values of body composition obtained by dual energy X-ray absorptometry in preterm and term neonates. J Pediatr Gastroenterol Nutr 1998;27:184-90.

8. Wrottesley SV, Pisa PT, Micklesfield LK, Pettifor JM, Norris SA. A comparison of body composition estimates using dual-energy X-ray 


\section{Articles | Dyke et al.}

absorptiometry and air-displacement plethysmography in South African neonates. Eur J Clin Nutr 2016;70:1254-8.

9. Dixon WT. Simple proton spectroscopic imaging. Radiology 1984;153: 189-94.

10. Yeung HN, Kormos DW. Separation of true fat and water images by correcting magnetic field inhomogeneity in situ. Radiology 1986;159: 783-6.

11. Glover GH, Schneider E. Three-point Dixon technique for true water/fat decomposition with B0 inhomogeneity correction. Magn Reson Med 1991;18:371-83.

12. Groves AM, Chiesa G, Durighel G, et al. Functional cardiac MRI in preterm and term newborns. Arch Dis Child Fetal Neonatal Ed 2011;96:86-91.

13. International Electrotechnical Commission Medical electrical equipment - part 2-33: particular requirements for the basic safety and essential performance of magnetic resonance equipment for medical diagnosis. IEC 60601-2-33:2010. 3.2, 2015-06.

14. Malik SJ, Beqiri A, Price AN, Teixeira JN, Hand JW, Hajnal JV. Specific absorption rate in neonates undergoing magnetic resonance procedures at 1.5T and 3T. NMR Biomed 2015;28:344-52.

15. Ward SR, Lieber RL. Density and hydration of fresh and fixed human skeletal muscle. J Biomech 2005;38:2317-20.

16. Martin AD, Daniel MZ, Drinkwater DT, Clarys JP. Adipose tissue density, estimated adipose lipid fraction and whole body adiposity in male cadavers. Int J Obes Relat Metab Disord 1994;18:79-83.
17. Ma J. Breath-hold water and fat imaging using a dual-echo two-point dixon technique with an efficient and robust phase-correction algorithm. Magn Reson Med 2004;52:415-9.

18. Harrington TA, Thomas EL, Frost G, Modi N, Bell JD. Distribution of adipose tissue in the newborn. Ped Res 2004;55:437-1.

19. Thomas EL, Saeed N, Hajnal JV, et al. Magnetic resonance imaging of total body fat. J Appl Physiol 1998;85:1778-85.

20. Ludwig UA, Klausmann F, Baumann S, et al. Whole-body MRI-based fat quantification: a comparison to air displacement plethysmography. J Magn Reson Imaging 2014;40:1437-44.

21. Bauer JS, Noel PB, Vollhardt C, et al. Accuracy and reproducibility of adipose tissue measurements in young infants by whole body magnetic resonance imaging. PLoS ONE 2015;10:1371.

22. Roggero $\mathrm{P}$, Giannì ML, Amato $\mathrm{O}$, et al. Is term newborn body composition being achieved postnatally in preterm infants? Early Hum Dev 2009;85:349-52.

23. Roggero $\mathrm{P}$, Giannì ML, Amato $\mathrm{O}$, et al. Evaluation of air-displacement plethysmography for body composition assessment in preterm infants. Pediatr Res 2012;72:316-20.

24. Uthaya S, Bell J, Modi N. Adipose tissue magnetic resonance imaging in the newborn. Horm Res 2004;62 (Suppl 3): 143-8.

25. Olhager E, Flinke E, Hannerstad U, Forsum E. Studies on human body composition during the first 4 months of life using magnetic resonance imaging and isotope dilution. Pediatr Res 2003;54:906-12. 